Legal barriers to access social housing in the commune of Santiago

\section{Barreras legales de acceso a la vivienda social en la comuna de Santiago*}

\author{
MICHELLE CÁCERES · Santiago, Chile·mcaceres@uchilefau.cl \\ Fecha de recepción: 15 de marzo 2019 - Fecha de aceptación: 30 de abril 2019
}

PALABRAS CLAVE • VIVIENDA SOCIAL · DEMANDA HABITACIONAL · SUBSIDIOS $\cdot$ SANTIAGO $\cdot$ GRUPOS VULNERABLES

KEYWORDS • SOCIAL HOUSING · HOUSINC

RESUMEN

El acceso formal a la vivienda social en Chile se ha consolidado principalmente como una política de financiamiento a una solución habitacional. Si bien es considerada exitosa, deja de lado a un porcentaje importante de la población, la que se encuentra con una serie de barreras o nudos estructurales al momento de postular y termina por no calzar en el perfil requerido para ser sujeto de beneficios. En este contexto, la comuna de Santiago, debido a su repoblamiento y crecimiento de las últimas décadas, es uno de los grandes focos de demanda habitacional y donde debido a sus características se dificulta acceder a vivienda social en la zona. El objetivo de este artículo es determinar por medio del análisis de los decretos DS.49 y DS.I cuáles son las barreras legales con las que se encuentran los habitantes al postular y cómo se expresan en la comuna de Santiago.

\section{ABSTRACT}

Formal access to social housing in Chile is mainly based on a financing policy for a housing solution. Although considered successful, it sets aside a significant percentage of the population, who face a series of barriers or structural knots when applying does not meet the requirements for said subsidy.

In this context, due to its repopulation and its growth in the last decades, the City of Santiago, is one of the great focuses of housing demand where, because of its characteristics, access to social housing in the area is difficult. The objective of this article is to determine, through the analysis of decrees DS.49 and DS.I, what the legal barriers are, that inhabitants face when applying and how they are expressed in the commune of Santiago. a política habitacional chilena es considerada - exitosa a partir de sus logros cuantitativos y la reducción del déficit habitacional a lo largo de los últimos años (Rodríguez \& Sugranyes, 2004). Al respecto, Ducci (2007) sostiene que para el 2007 ya se estimaba que únicamente el $15 \%$ de la población total del país no poseía una vivienda adecuada y que los asentamientos irregulares constituían solo el $2 \%$ del catastro total del país. Estas cifras resultan altas en comparación con otros países de la región que tienen alrededor del $30 \%$ o $40 \%$ de sus habitantes en condiciones de irregularidad (Ducci, 2007),

A pesar de las exitosas cifras, el sistema de acceso a la vivienda chileno se ha concentrado principalmente en la adquisición del bien físico, lo que comenzó a mostrar las primeras dificultades y problemáticas para sus usuarios en el año 1997, como bien resume Casgrain: "Algunos autores han identificado problemas que derivan directamente de la construcción masiva de viviendas sociales, problemas concernientes a la calidad de la vivienda construida, la segregación socioespacial y el endeudamiento de los 'beneficiarios'" (Casgrain, 2010, p. 157). El éxito del sistema se basa en la intervención de privados, quienes construyen barrios completos en los paños más económicos de la periferia de la ciudad, potenciando su crecimiento horizontal y expansión urbana no controlada; todo esto concluyendo en gran insatisfacción residencial, altos niveles de morosidad en pagos y un aumento en el del déficit cualitativo (Rodríguez \& Sugranyes, 2004).

Si bien el sistema de subsidios chileno ha realizado importantes cambios en la política habitacional a partir del 2006 con la promoción de construcción de unidades más grandes, aumento del monto del subsidio, introducción de un subsidio de localización y el inicio del programa de recuperación integral de barrios, su principal foco de acceso a la vivienda sigue siendo el financiamiento del inmueble físico, dejando de lado a un porcentaje importante de la población que ya sea por no calzar en el perfil de postulante o por no parecerle lo suficientemente atractiva la oferta existente (por ejemplo, no hay oferta de vivienda en su misma comuna), termina por convertirse en una persona "no hábil", es decir, no sujeta a los beneficios que pudiese entregarle el Estado en términos de subsidios. Uno de los tantos reflejos de esta situación se ve plasmado en el aumento sostenido del allegamiento y del déficit habitacional estimado en 497.560 unidades (CASEN, 2017), sumados a la existencia de una demanda acumulada de subsidios sin proyecto habitacional, cifra estimada en unos 38.915 (Pizarro, 2016) subsidios al 2016.

En este contexto, la comuna de Santiago, tercera más poblada del país, debido a su ubicación central y por ser uno de los focos

\footnotetext{
* Artículo realizado a partir de los resultados del seminario de investigación homónimo, realizado por la autora en su proceso de formación profesional (Profesora Guía: Paola Jirón).
} 
principales de empleo y equipamiento; es uno de los grandes focos de demanda habitacional a nivel país. Prueba de esto es el aumento de su población en un 55,09\% (Municipalidad de Santiago, 2015) entre los años 2002-2012, revirtiendo así el despoblamiento del sector como resultado del conjunto de iniciativas del Municipio que incluyeron la creación de la Corporación de Desarrollo de Santiago (Jirón, 1999). Sin embargo, debido al alto valor del precio del suelo en esta comuna y la escasez de este, es prácticamente imposible costear proyectos de vivienda social con el bajo monto del subsidio habitacional. Ante un aumento sostenido de la demanda, las barreras ya existentes por decreto para acceder a la vivienda social se suman a las complejidades propias del territorio de una comuna consolidada y antigua.

Debido a esta situación, este artículo analiza los distintos impedimentos legales o barreras con los que se encuentran los habitantes al postular a un subsidio habitacional, y cómo estas se expresan en el caso de la comuna de Santiago. Como metodología principal se utilizó el análisis de los decretos DS.49 (Reglamento del programa fondo solidario de elección de vivienda) y DS. 1 (Reglamento del sistema integrado de subsidio habitacional), los cuales son los principales mecanismos de acceso a la vivienda para grupos vulnerables por vía formal del Estado, lo que se contrastó con fuentes de información vinculada a bibliografía y documentos asociados.

\section{BARRERAS DE ACCESO A LA VIVIENDA SOCIAL}

Actualmente los habitantes de grupos vulnerables se encuentran con diferentes limitaciones para acceder a la casa propia por medio de los subsidios de la política habitacional chilena. Estas limitaciones están determinadas por los decretos que regulan el sistema de postulación a subsidios, a lo que se suman los problemas adicionales de cada territorio. Para conocer cuáles son estas limitaciones, se analizan dos de los principales subsidios que utilizan los habitantes de sectores vulnerables para acceder a la vivienda: El Fondo solidario de elección de vivienda o DS.49 y el subsidio para sectores medios o DS. 1 .

\section{SUBSIDIO DS.49}

Este subsidio, focalizado en los grupos vulnerables que estén dentro del $40 \%$ de la población con menos recursos del país, pretende financiar una vivienda nueva o usada de hasta 950 UF, sin crédito hipotecario en zonas urbanas o rurales (Ministerio de Vivienda y Urbanismo, 2019). En su decreto, se identifican las siguientes barreras:

- El ahorro. Se requieren 10 UF mínimas para la postulación a la vivienda básica. Este requisito es el primer impedimento con el que se topan las familias, considerando que suelen tener ingresos esporádicos y una situación laboral inestable.

- Haber sido asignatario de una "solución habitacional". Esta barrera explicita que cualquier integrante del grupo familiar que sea o haya sido propietario de una caseta sanitaria, vivienda, o sitio (DS.N49, 2017) queda inhabilitado para postular. A pesar de que la política pública apunta a no focalizar recursos donde ya hayan sido inyectados, muchas familias no poseen soluciones adecuadas a sus necesidades y viven en altas condiciones de vulnerabilidad a pesar de haber obtenido, por ejemplo, la caseta sanitaria.

- Pertenecer a un grupo familiar. No pueden postular las personas que no acrediten pertenencia a un grupo familiar. Salvo las siguientes excepciones: Personas que acrediten discapacidad; con condición de viudez; con ascendencia indígena; y quienes hayan sido víctimas de tortura o exilio. (DS.N49, 2017). Lo anterior complica principalmente a los solteros de grupos vulnerables, dificultándoles e imposibilitándoles la independencia de su grupo familiar, el que suele haber sido asignatario previo de una solución habitacional.

- Tenencia acreditada de sitios. Para la construcción en sitio propio, resulta obligatorio acreditar la propiedad del terreno (DS.N49, 2017). La mayoría de los sitios de personas de grupos vulnerables han sido obtenidos en condiciones de irregularidad, o poseen problemas de sucesión, ya que al heredar no se hizo el trámite correctamente. Esto los imposibilita para postular a cualquier programa de densificación o mejoramiento de vivienda.

\section{- Falta de acceso a una Entidad} Patrocinante (EP). A pesar de que esta barrera de acceso se encuentra implícita en el decreto, se especifica que la postulación de los beneficiarios actualmente es gestionada por EP, las que pueden ser los municipios o agentes privados. Lamentablemente existen una serie de comunidades que no pueden acceder a este tipo de gestión, ya sea por temas económicos, aislamiento o incluso falta de información.

- Falta de acceso a información. En caso de no contar con el conocimiento o acceso a información sobre las ayudas del Estado para la postulación a soluciones habitacionales, los grupos de potenciales beneficiarios ni siquiera serán considerados como demanda efectiva al no elevar una solicitud.

\section{SUBSIDIO DS. 1}

Este subsidio se focaliza en los sectores medios para financiar la compra de una vivienda nueva o usada complementándola con recursos propios o crédito hipotecario. Ofrece tres tramos distintos de postulación: Título I tramo 1 para una vivienda de máximo 1.000 UF y ahorro mínimo de 30 UF; Título I tramo 2 para una vivienda de máximo 1.400 UF y un ahorro de 40 UF y título II para una vivienda de máximo 2.200 UF y un ahorro de 80 UF. (Ministerio de Vivienda y Urbanismo, 2019b). 


\section{BARRERAS DE ACCESO A LA VIVIENDA DEL SUBSIDIO PARA FAMILIAS DE SECTORES MEDIOS}

Respecto a las barreras o nudos estructurales encontrados en el DS. 1, la primera diferencia que se vislumbra en los títulos del decreto es que, a diferencia del DS. 49, en este sí pueden postular solteros. A pesar de ello, se repiten algunas dificultades y aparecen otras nuevas que se conjugan de la siguiente manera:

- Altos montos de ahorro. El monto mínimo para postular al primer título del DS.1, son 30 UF, pudiendo llegar en el título III a las 80 UF como mínimo, lo que además se complementa con un crédito hipotecario debido a los altos costos de la vivienda a la que se apunta (DS. $\left.N^{\circ} 1,2011\right)$.

- Tenencia acreditada de sitios. Al igual que en el DS. 49, se puede postular a la densificación en sitio propio o construcción en él, siempre y cuando esté acreditada la posesión del terreno. En esta dificultad incurren muchas personas en situación de vulnerabilidad, donde a pesar de ser propietarios no se ha hecho correctamente la sucesión del terreno, o se ha obtenido de manera irregular.

- Haber sido asignatario de una "solución habitacional". Al igual que en el DS.49, el haber sido asignatario de cualquier solución habitacional, ya sea subsidio, caseta sanitaria, un abono municipal, etc. inhabilitará al postulante, salvo que la solución que haya obtenido haya resultado destruida o deficiente.

- Estigmatización social. La clasificación socioeconómica que realiza el DS.1, al no tener un mínimo de ingresos, posibilita a habitantes de los sectores más vulnerables a postular a él, sin tener la capacidad de ahorro ni de endeudamiento de otros grupos socioeconómicos. Al postular, se los encasilla automáticamente en "grupos medios" lo que marca una diferencia social con respecto a una postulación a DS.49, lo que obliga al habitante a reconocerse como "sujeto vulnerable".

- Acceso a información. Al igual que en el DS.49, los posibles asignatarios de beneficios son quienes efectivamente elevan una solicitud, por tanto al no tener la información disponible o el conocimiento sobre los beneficios del Estado, no pueden postular.

\section{ANÁLISIS COMPARADO DE BARRERAS DE ACCESO EN DECRETOS DS. 1 Y DS.49}

Al analizar ambos subsidios aparecen distintos requerimientos que complejizan la postulación de los beneficiarios, los que, como puede verse en la TABLA 1, resultan muy similares en ambos casos.

Tabla 1. Cuadro comparativo barreras en DS. 1 Y DS.49

\begin{tabular}{|c|c|c|}
\hline Barrera de acceso & DS.49 grupos vulnerables & DS. 1 grupos medios \\
\hline Asociada al ahorro & $\begin{array}{l}\text { Mínimo ahorro } 10 \text { UF, lo que deja fuera a todos los } \\
\text { grupos familiares que no tienen capacidad de ahorro } \\
\text { efectiva al presentar muy bajos ingresos e irregularidad } \\
\text { de las fuentes de trabajo. }\end{array}$ & $\begin{array}{l}\text { "Mínimo ahorro } 30 \text { UF. } \\
\text { Para tramos } 2 \text { y 3: preaprobación de crédito hipotecario, } \\
\text { si no, acreditar ahorro mínimo de } 200 \text { UF en tramo } 2 \text { y } \\
400 \text { UF en tramo } 3 \text {. }\end{array}$ \\
\hline Composición familiar & Mínimo 2 integrantes por grupo familiar. & No tiene. \\
\hline Tenencia legal de sitio & $\begin{array}{l}\text { Solo para modalidad de construcción en sitio propio } \\
\text { individual, en la modalidad colectiva todas requieren } \\
\text { propiedad efectiva, si no es en propiedad, en proceso } \\
\text { de compra demostrable. }\end{array}$ & $\begin{array}{l}\text { Acreditación de propiedad efectiva necesaria para la } \\
\text { postulación en la modalidad de "construcción en sitio } \\
\text { propio". }\end{array}$ \\
\hline Asignación previa de beneficio habitacional & $\begin{array}{l}\text { El grupo queda inhabilitado si cualquiera de sus } \\
\text { integrantes ya fue asignatario de solución habitacional. }\end{array}$ & $\begin{array}{l}\text { El grupo queda inhabilitado si cualquiera de sus } \\
\text { integrantes ya fue asignatario de solución habitacional. }\end{array}$ \\
\hline Estigmatización social & $\begin{array}{l}\text { Al ser asignatario, el grupo familiar queda } \\
\text { inmediatamente encasillado como grupo vulnerable, } \\
\text { en otras palabras, se reconoce a sí mismo como una } \\
\text { persona de escasos recursos. }\end{array}$ & Al ser asignatario, se reconoce como clase media. \\
\hline Acceso a Entidad Patrocinante (EP) & El acceso a una EP es requisito para la postulación. & $\begin{array}{l}\text { En el caso de las postulaciones colectivas, igualmente es } \\
\text { un requerimiento postular a través de una EP. }\end{array}$ \\
\hline Acceso a información & $\begin{array}{l}\text { En este caso, la postulación es inexistente, ya que el } \\
\text { posible beneficiario no se entera de la existencia de } \\
\text { los beneficios por la manera en que opera la política } \\
\text { habitacional (por demanda efectiva). }\end{array}$ & $\begin{array}{l}\text { En este caso, la postulación es inexistente, ya que el } \\
\text { posible beneficiario no se entera de la existencia de } \\
\text { los beneficios por la manera en que opera la política } \\
\text { habitacional (por demanda efectiva). }\end{array}$ \\
\hline
\end{tabular}

Fuente: Elaboración propia en base a DS.1, DS.4 


\section{BARRERAS DE ACCESO EN LA COMUNA DE SANTIAGO}

La comuna de Santiago, con 404.495

habitantes según el Censo 2017, es el punto de convergencia de una serie de características que aumentan la dificultad al momento de acceder a la vivienda en la comuna para los grupos vulnerables. Construcciones antiguas, el valor del suelo, la escasez de este, los tamaños y morfología de los terrenos hacen de la comuna un lugar emblemático donde las posibilidades de costear una vivienda social con el monto del subsidio son muy bajas.

Las barreras existentes por parte de la política habitacional identificadas y que se presentan de manera crítica en la comuna son las siguientes:

\section{TENENCIA ACREDITADA DE SITIOS}

Esta es una de las barreras más complejas al momento de acceder a la vivienda social en la comuna, pues convergen varios factores: La antigüedad de las construcciones y la existencia de múltiples propiedades sin saneamiento legal y dueños inubicables. Esta situación que termina por inhabilitar las postulaciones a subsidios de construcción en sitio propio, densificación predial y prácticamente todas las modalidades colectivas, se agrava debido a la existencia de numerosas ampliaciones irregulares en la comuna y proyectos de autoconstrucción con más de 100 años de antigüedad, que no pueden postular a los subsidios DS.49 y DS. 1 ni a los otros programas existentes de mejoramiento en la comuna al no contar con la tenencia acreditada.

\section{INEXISTENCIA DE PROYECTO HABITACIONAL}

Entre las principales dificultades para acceder a la vivienda en la comuna se encuentran la escasez de suelo y el encarecimiento progresivo de este. Esta situación termina por agravar y dificultar las posibilidades de acceso a la vivienda en la comuna para nuevos y antiguos residentes, pues complejiza la capacidad de efectuar proyectos de construcción de vivienda (sobre todo proyectos de subsidio DS.49, teniendo en cuenta que el subsidio pretende cubrir el costo del terreno y la construcción), convirtiéndose efectivamente en una barrera pues las postulaciones se reducen o se anulan por la inexistencia de proyectos.

Lo anterior se complementa a la existencia de una ciudad y comuna consolidadas: las características del suelo también afectan y aumentan el valor de la construcción. La morfología de los pocos terrenos a disposición es en muchos casos de frentes angostos e irregulares, con entornos con construcciones preexistentes, adosamientos, fachada continua, entre otros, lo que termina por encarecer más aún la ejecución de los proyectos, la que claramente presenta diferencias sustanciales en montos respecto a la construcción en periferia donde los terrenos no tienen mayores complejidades.

Por otro lado, la oferta existente de subsidios DS. 1 se configura principalmente a través de un tipo de propuesta: departamentos de $1 \circ 2$ dormitorios, lo que no abarca necesariamente las necesidades de la población vulnerable y tiende a ser absorbida por otro tipo de población como afirma Arriagada: "El producto para las clases medias es para las clases medias no residentes interesados en vivir cerca de su trabajo o la universidad (...) son gente que quiere hacer una inversión(...) la oferta de renovación urbana que hubo fue muy masiva, pero fue masiva con ciertas tipologías habitacionales de 1 - 2 dormitorios, por tanto no estaban pensadas para familias, estaban pensadas para sujetos y para parejas lo que no es la realidad de los pobladores históricos del centro o pericentro" (Arriagada, comunicación personal, 27 de mayo 2016).

\section{COMPOSICIÓN FAMILIAR}

Otro de los requerimientos que terminan por inhabilitar a los postulantes en el caso del DS.49 en la comuna de Santiago es la necesaria pertenencia a un grupo familiar (postular con más de 2 integrantes). La realidad de las familias en las comunas centrales, donde muchas de ellas son propietarias o ya han sido asignatarias de alguna solución habitacional, es que cuando sus hijos son solteros y se encuentran en condiciones de vulnerabilidad -en general, allegados- no pueden postular al subsidio por no integrar un grupo familiar.

Este requisito marca una diferencia respecto a la oferta que presenta el DS. 1, que no exige la pertenencia a un grupo familiar: Por ser vulnerable, no puedo postular solo. Esta realidad que termina por construir la política pública es compleja al momento de abrir oportunidades a grupos emergentes, pues como se reparten los recursos por medio de puntaje, los solteros o familias muy pequeñas no tienen oportunidad, tal y como especifica Larenas: "Una barrera de acceso para optar al Ds. 49, es que siendo soltero no tienes ninguna posibilidad porque se privilegian a las familias. Además, en la condición que establece el Ds. 49 que tienes un solo programa de vivienda, una vivienda o departamento de 55 m2, un baño, cocina, 3 dormitorios, o sea sin ninguna flexibilidad ni para arriba, ni para abajo. Una familia numerosa no tiene ninguna posibilidad de vivir adecuadamente bajo el marco normativo del Ds. 49 y un sujeto solo, tampoco, porque 'le sobra' espacio, y no es foco de la política, porque es un tipo que si vive de allegado, no es un problema" (J. Larenas, comunicación personal, 10 de mayo 2016).

\section{ASIGNATARIOS PREVIOS DE UNA SOLUCIÓN HABITACIONAL}

Otro de los requisitos asociados al DS.49 y DS. 1 que se traducen en una barrera estructural y con altas repercusiones en la comuna de Santiago, es ser asignatario previo de una solución habitacional. La realidad de una comuna con la antigüedad de Santiago, sumado a la necesaria postulación con un grupo familiar al subsidio de grupos vulnerables conlleva a que existan 
habitantes que se ven inhabilitados y terminan viviendo de allegados. Estas dos barreras suelen presentarse de manera conjunta en la comuna, dejando fuera a todos quienes hayan quedado "marcados" por la asignación, pues si la oportunidad para postular era asociarse a uno de sus familiares, lo común es que ellos ya hayan sido asignatarios de una solución habitacional.

\section{NACIONALIDAD Y RESIDENCIA DEFINITIVA EN CHILE}

Otro nudo estructural para una comuna que posee más del $28 \%$ de su población compuesta por inmigrantes (Censo, 2017) es la necesaria posesión de nacionalidad y residencia definitiva en Chile.

De esta manera, uno de los grupos más afectados en la comuna son los inmigrantes, cifra estimada en el $54,4 \%$ del total regional (Segura y Abde, 2014), siendo Santiago la comuna que más inmigrantes concentra de toda la Región Metropolitana. En este grupo convergen todas las dificultades con las que ya se encuentran los "chilenos" de la comuna, más la barrera asociada a la ilegalidad y las diferencias y estigmatizaciones culturales. Es más, mientras no logran acceder, se encuentran con un mercado informal de vivienda de precarias condiciones que terminan por complejizar aún más su situación de habitabilidad y vulnerabilidad.

\section{DISCUSIÓN DE RESULTADOS: LAS ALTERNATIVAS}

Con la existencia de una política habitacional restrictiva y compleja de aplicar en comunas consolidadas como Santiago, los vecinos han optado por diversas alternativas de acceso informal a la solución habitacional: el allegamiento, tomas de terreno, el acceso autogestionado y el arrendamiento en mercado informal. En tanto a las alternativas en el caso de Santiago, los más críticos identificados en esta investigación, son el allegamiento y el mercado de arrendamiento informal que existe en la comuna, ambos con marcados rasgos de precariedad, vulnerabilidad y riesgo asociados.

El arriendo informal suele ser la estrategia más utilizada por parte de los inmigrantes, en cambio, el allegamiento es asociado a antiguos residentes con o sin subsidio y grupos unifamiliares que no pueden acceder al DS.49. Por otro lado, los adultos mayores, son de los más afectados al no poseer redes de apoyo y estar comúnmente marcados por la barrera que implica el haber obtenido ya una solución habitacional. Por ello, suelen tener la única alternativa de vivir en la vivienda o solución que ya les fue asignada, o acudir a ONG'S alternativas en búsqueda de una vivienda que se adapte mejor, como es el caso de la Fundación Vivienda: "muchos de ellos llegan a la fundación a buscar una mediagua o una vivienda de emergencia progresiva" (A. Rivas, comunicación personal, 7 de junio 2016).

Por el lado institucional, las complicaciones de acceso a la vivienda en la comuna han sido identificadas como un problema por parte del Municipio de Santiago. De esta manera, se ha asumido la escasez de suelo y el precio de este como impedimentos para que se construya vivienda social en la comuna debido a los altos costos asociados. A partir de esto se han elaborado una serie de estrategias que apuntan a generar un camino alternativo que continúe trabajando con los subsidios ministeriales y al mismo tiempo, convertir en sujetos hábiles a quienes no son sujetos de subsidio. Estas estrategias se han concretado en la gestión y compra de suelo para separar así el costo del terreno de la construcción, logrando dedicar el subsidio ministerial completo a la construcción de vivienda.

Aun así, como se mencionó anteriormente, la calidad de los terrenos disponibles suelen ser retazos con morfologías complejas y difíciles de abordar, con condiciones que dejan al subsidio totalmente desvalorizado respecto a una construcción en un terreno uniforme y sin construcciones preexistentes alrededor, lo que también termina por ser poco atractivo para las constructoras por los mencionados costos asociados y consecuente disminución de la rentabilidad del proyecto.

\section{CONCLUSIONES}

Son conocidas las consecuencias de una política habitacional enfocada únicamente en la provisión de una solución física y sin considerar las particularidades de su entorno. Como han identificado diversos autores, la producción masiva de vivienda social bajo el modelo subsidiario ha consolidado bolsones de pobreza en la periferia, construido vivienda de mala calidad y creado problemas de equipamiento y acceso a la ciudad (Ducci, 1997; Sabatini, Cáceres y Cerda, 2001 ; Sepúlveda et al., 2009; Simian, 2010; Rodriguez \& Sugrayes, 2011 1). Lo anterior, tal y como identifican Castillo \& Forray (2014), se debe a que el principal problema de acceso a la vivienda es el financiamiento de suelo bien localizado, cuyo valor es muy alto. Debido a que el monto del subsidio habitacional pretende cubrir el costo de suelo y la construcción de la vivienda, esto obliga a construir en los paños más económicos de la ciudad, resultando prácticamente imposible para los asignatarios de beneficios del Estado conseguir una solución de vivienda en una comuna consolidada como Santiago.

En un contexto en el que tener proyectos de vivienda social en la propia comuna ya es un tema complejo, se suman las dificultades propias de la comuna, pues el suelo además de caro, es escaso, irregular y rodeado de una ciudad consolidada. Por si esto fuera poco, además de estas dificultades, los habitantes se encuentran con la serie de barreras legales descritas en el artículo que vuelven aún más difícil el acceso a una solución habitacional por las vías formales.

Ante este panorama, las alternativas de los habitantes para residir en la comuna se transforman prácticamente en la única opción viable para solucionar sus necesidades 
habitacionales, dando pie a un amplio mercado de arriendo informal, altos niveles de allegamiento y hacinamiento.

Esta desactualización de la política habitacional que les impide a postulantes en condición de vulnerabilidad acceder a una solución habitacional es la consecuencia de una hiperfocalización que no logra adaptarse a las particularidades de los territorios. El hecho de que existan habitantes vulnerables que quedan fuera por no cumplir con los requerimientos automáticamente aumenta su nivel de vulnerabilidad, pues sumado a su imposibilidad de resolver sus necesidades por sí mismos, además cuentan con una serie de impedimentos para ser auxiliados por las ayudas estatales.

Por otro lado, tal y como plantea Fuster-Farfán (2019), las distintas soluciones ejemplares de vivienda social en comunas centrales que existen como alternativa a la segregación y expulsión a la periferia de los habitantes de la comuna, no son más que casos aislados que no representan la norma general de la producción habitacional en Chile. Nos encontramos ante una situación compleja que para ser adaptada a las necesidades de un territorio, depende exclusivamente de la capacidad e interés de los agentes locales, su prioridad política y los diversos contextos en los que se encuentran (Fuster-Farfán, 2019).

De esta manera nos encontramos en un momento crítico de la política habitacional chilena, donde las soluciones deben plantearse de manera situada a la realidad nacional y los modos de habitar de nuestros residentes. Este estudio pretende mostrar los diferentes puntos críticos que plantea la ley misma por medio de los decretos DS.49 Y DS. 1 de acceso a la vivienda, que terminan por actuar como una camisa de fuerza para los habitantes. Una vez identificados, es posible aportar a las futuras o potenciales modificaciones y contextualizarlas en la realidad actual de los habitantes de nuestro territorio.

\section{REFERENCIAS BIBLIOGRÁFICAS}

Casgrain, A. (2010). La apuesta del endeudamiento en política habitacional chilena. Revista Invi, 25(68), 155-182.

Castillo, M. J., \& Forray, R. (2014). La vivienda, un problema de acceso al suelo. ARQ (Santiago), (86), 48-57. Recuperado de https://dx.doi. org/10.4067/S0717-69962014000100007

Ds N 49 Aprueba reglamento del programa Fondo Solidario de elección de vivienda. Diario oficial de la República de Chile. Santiago 21 de junio de 2017

DS. $N^{\circ} 1$ Aprueba reglamento del sistema integrado de subsidio habitacional. Santiago: Diario oficial de la República de Chile. Santiago 27 de diciembre de 2011.

Ducci, M. E. (1997). Chile: El lado oscuro de una política de vivienda exitosa. EURE, 99-115.

Ducci, M. E. (2007). "La política habitacional como instrumento de desintegración social. Efectos de una política de vivienda exitosa". En R. H. María José Castillo, 1906-2006. Cien años de política de vivienda en Chile (págs. 107-123). Santiago: Universidad Andrés Bello.

Fuster-Farfán, X. (2019). Las políticas de vivienda social en Chile en un contexto de neoliberalismo híbrido. Revista EURE - Revista De Estudios Urbano Regionales, 45(135).

Instituto Nacional de Estadísticas (2018) Resultados definitivos del Censo 2017. Santiago: Instituto Nacional de Estadísticas.

Jirón, P. (1999). Planificación y gestión estratégica en la comuna de Santiago. ¿̇Son las herramientas o los objetivos inapropiados? INVI, 14(36), 105 118.

Ministerio de Desarrollo Social (2018). Encuesta de caracterización socioeconómica nacional CASEN 2017. Santiago: Ministerio de Desarrollo Social.
Ministerio de Vivienda y Urbanismo (2019). Comprar una vivienda. Fondo Solidario de Elección de vivienda. Ministerio de Vivienda y Urbanismo. Recuperado de https://beneficios.minvu.gob. $\mathrm{cl} /$ comprar-una-vivienda/sector-vulnerable/ accedido el accedido el 5-03-2019.

Ministerio de Vivienda y Urbanismo (2019b). Comprar una vivienda. Sectores medios. Ministerio de Vivienda y Urbanismo. Recuperado de https:// beneficios.minvu.gob.cl/comprar-una-vivienda/ sectores-medios/ accedido el accedido el 5-032019.

Municipalidad de Santiago (2015). Política de promoción de vivienda inclusiva. [Diapositivas de PowerPoint]. s.f

Pizarro, A. (2016). Conferencia de SERVIU metropolitano realizada en la Facultad de Arquitectura y Urbanismo de la Universidad de Chile: Inauguración del año académico de escuela de Postgrado, 1 de abril 2016.

Rodríguez, A., \& Sugranyes, A. (2004). El problema de la vivienda de los "Con Techo". EURE, 53-65.

Rodríguez, A., \& Sugranyes, A. (2011). Vivienda privada de ciudad. Revista de Ingeniería, (35), 100-107. Recuperado de http://www.scielo.org. co/scielo.php?script=sci_arttext\&pid =S0121 $49932011000300016 \&$ Ing =en\&tlng=es.

Sabatini, F., Cáceres, G., \& Cerda, J. (2001). Segregación residencial en las principales ciudades chilenas: tendencias de las tres últimas décadas y posibles cursos de acción. Revista EURE - Revista De Estudios Urbano Regionales, 27(82).

Segura, D. M., \& Abde, K. B. (2014). Barrios y población inmigrantes: el caso de la comuna de Santiago. INVI, 19-77.

Sepúlveda, R., Larenas, J., Prado, V., Prat, B., \& Álvarez, J. (2009). Bicentenario: Oportunidad de repensar las políticas urbano-habitacionales en Chile. Revista INVI, 24(67), 21-67

Simian, J. M. (2010). Logros y desafíos de la política habitacional en Chile. Estudios públicos, 269 320 . 